hep-th/0202210

HUTP-02/A001

\title{
Spacelike Branes
}

\author{
Michael Gutperle and Andrew Strominger \\ Jefferson Physical Laboratory \\ Harvard University \\ Cambridge, MA 02138
}

\begin{abstract}
Scalar field theories with appropriate potentials in Minkowski space can have timedependent classical solutions containing topological defects which correspond to S-branes - i.e. branes all of whose tangential dimensions are spacelike. It is argued that such Sbranes arise in string theory as time-dependent solutions of the worldvolume tachyon field of an unstable D-brane or D-brane-anti-D-brane pair. Using the known coupling of the spacetime RR fields to the worldvolume tachyon it is shown that these S-branes carry a charge, defined as the integral of a RR field strength over a sphere (containing a time as well as spatial dimensions) surrounding the S-brane. This same charge is carried by SDbranes, i.e. Dirichlet branes arising from open string worldsheet conformal field theories with a Dirichlet boundary condition on the timelike dimension. The corresponding SDbrane boundary state is constructed. Supergravity solutions carrying the same charges are also found for a few cases.
\end{abstract}




\section{Contents}

1. Introduction . . . . . . . . . . . . . . . . . . . . . . . . . . . . . . . . . 1

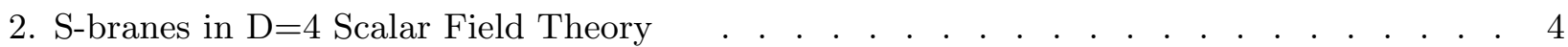

2.1. An S2-brane Domain Wall . . . . . . . . . . . . . . . . . . . . . . . . . . 4

2.2. An S1-brane Vortex . . . . . . . . . . . . . . . . . . . . . . . . . . . . 5

2.3. Charged S-branes . . . . . . . . . . . . . . . . . . . . . . . . 6

2.4. R-symmetry . . . . . . . . . . . . . . . . . . . . . . . . 8

3. S-branes in String Theory . . . . . . . . . . . . . . . . . . . . . . 8

3.1. S-branes as Tachyon Solitons . . . . . . . . . . . . . . . . . . . . . . . . 9

3.2. S-branes within Branes as Evanescent Sphalerons . . . . . . . . . . . . . . . . 10

3.3. NS S-branes . . . . . . . . . . . . . . . . . . . . . . . . . . . . . . . . 11

4. Spacelike D-branes . . . . . . . . . . . . . . . . . . . . . . . . . . . . . . 11

4.1. The Boundary State . . . . . . . . . . . . . . . . . . . . . . . . . . . 12

4.2. Discussion . . . . . . . . . . . . . . . . . . . . . . . . . . . . . . . . . 14

5. Spacetime Solutions . . . . . . . . . . . . . . . . . . . . . . . 15

5.1. An S0-brane in $\mathrm{D}=4$. . . . . . . . . . . . . . . . . . . . . . . . . . . . 16

5.2. An S5-brane in $\mathrm{D}=11$. . . . . . . . . . . . . . . . . . . . . . . . . . . . 17

6. Singularities . . . . . . . . . . . . . . . . . . . . . . . . . . 19

7. Summary . . . . . . . . . . . . . . . . . . . . . . . . . . . . . . . 20

\section{Introduction}

In this paper it is argued that string theory - as well as many field theories - contain time dependent solutions corresponding to spacelike branes or S-branes. These are topological defects which are localized on a spacelike hypersurface and hence exist only for a moment in time. It is further suggested that some of these defects are related to spacelike versions of the familiar D-branes [1] (or SD-branes), in which the time coordinate obeys a Dirichlet boundary condition. The main piece of evidence for this proposed relation is that they carry the same type of RR charge. However, as discussed herein, a detailed analysis indicates possible subtleties in the relationship. Similar arguments suggest the existence of branes on null hypersurfaces or N-branes.

Interest in such configurations arises from several related viewpoints. Over the past several decades an astonishing and rich variety of phenomena have been discovered in string theory which have altered our fundamental notion of space. Virtually all of these phenomena concern static geometries. In general relativity, space and time are on the same footing, so we should expect equally surprising phenomena in the time-dependent context. In order to find these phenomena we should study time-dependent string backgrounds. 
Flat spacetimes with a Dirichlet boundary condition in the time direction (an SD-brane) or null direction (ND-brane) are some of the simplest imaginable time dependent conformal field theories.

One of the concepts one hopes to generalize from a spatial to a temporal context is holography. In the AdS/CFT correspondence [2-4], the D-brane field theory holographically reconstructs a spatial dimension. By analogy, SD-branes should holographically reconstruct a time dimension. Such a temporal reconstruction was argued to be a key ingredient of a proposed dS/CFT correspondence [547] (see also [8,9]). Indeed the present work was in large part motivated in an attempt to understand how string theory might produce the Euclidean field theories required for a dS/CFT correspondence. S-branes might also be relevant for the resolution of the spacelike singularities inside black holes.

The basic idea - illustrated by a specific example - is as follows. Type IIA string theory contains an unstable D3-brane with a tachyon field whose potential resembles a double well. Sen [10] has argued the the stable D2-brane is the tachyonic kink solution of the unstable D3 worldvolume field theory. Now consider initial data at time $t=0$ for the D3 with the tachyon field perched at the unstable minimum and given a small constant positive velocity. As time evolves into the future, the tachyon will roll off the top, emit closed string radiation, and eventually settle in to the positive minimum. Evolving into the past from $t=0$, one finds the time reversed picture with the tachyon approaching the negative minimum at $t=-\infty$. The full picture consists of finely tuned incoming radiation which conspires to excite the tachyon field to the top of the potential barrier. The tachyon then rolls down the other side and dissipates its energy back in to radiation. The result is a timelike kink in the tachyon field which is an S2-brane. $\mathrm{E}$ A similar discussion involving initial data on null hypersurfaces leads to N-branes (see [11]).

Using the known couplings of the open string tachyon to the RR fields, one concludes that this configuration carries the same kind of charge 2 as that carried by a spacelike D2brane. In analogy with Sen's identification of the spacelike kink as an ordinary D2-brane, this motivates the identification of the timelike kink as an SD2-brane.

1 We adopt conventions such that an Sp-brane has $\mathrm{p}+1$ spatial dimensions and no time dimensions.

2 This charge is measured by an integral of the RR 6-form over the $S^{6}$ surrounding the brane. This $S^{6}$ has both timelike and spacelike dimensions. It is perhaps an abuse of terminology to refer to such an integral as a charge because it is not time independent, but we nevertheless find it convenient. 
This construction has obvious generalizations to other (co)dimensions, as well as to branes as vortices in brane-antibrane pairs, etc. Furthermore in analogy to the description of branes within branes as instantons [12], S-branes within branes can be described as decaying sphaleron type configurations. In fact in line with the discussion of [13] this might be related to the tachyon soliton description of branes.

In analogy with AdS/CFT, one anticipates that the worldvolume field theory on a stack of $N$ S-branes is holographically dual to the supergravity solution sourced by the S-branes. As a small step in investigating such a possible connection, we find two examples of such spacetime solutions.

This paper is organized as follows. In section 2.1 a real scalar field in four spacetime dimensions with a double well potential is considered, and argued to have a timelike kink which corresponds to an S2-brane. Section 2.2 considers a complex scalar with a Mexican hat potential and describes the S1-brane solution. In 2.3 three-form and two-form field strengths are coupled to S1-brane and S0-brane solutions, and the corresponding charges and gauge field configurations are discussed. In section 2.4 the R-symmetry group of Lorentz transformations which leave the S-brane location fixed is discussed. This is argued to be broken, possibly down to spatial rotations, at scales of order the brane thickness. Section 3.1 embeds this discussion in string theory using the fact that the worldvolume tachyon in an unstable D-brane (D-brane-anti-D-brane pair) has a double well (Mexican hat) potential. In section 3.2 the discussion is generalized to S-branes within branes. Time dependent D3-brane worldvolume configurations are described (following [14]) which have a nonzero value of $\int \operatorname{tr} F \wedge F$ which is localized in time. These carry $R R$ charge corresponding to an SD(-1)-brane. Section 3.3 briefly mentions the generalization to NS charges. In section 4 a description of these S-branes as Dirichlet branes is considered. In section 4.1 the boundary state for a D-brane with Dirichlet boundary conditions on a timelike coordinate is constructed. Unlike the usual case, the SD-brane boundary state has an on-shell component. This represents the initial close strings which converge and create the SD-brane and the outgoing closed strings which represent its decay products. The corresponding classical closed string field configuration is found to have singularities along the past and future light cones of the brane. These arise (as for ordinary branes) because in the leading order description the closed strings are squeezed into a zero-width region along the brane. The $\mathrm{RR}$ charge associated to a sphere surrounding the S-brane is computed from the boundary state. In section 4.2 the problem of how the incoming closed strings in the boundary state "know" to make an SD-brane is discussed. We also present 
a puzzle concerning the brane energy density, for which the soliton and D-brane pictures do not obviously agree, and which indicates subtleties in the relationship between the two. The worldvolume theory of the SD-brane is also briefly discussed. Section 5 contains a preliminary discussion of the classical (super)gravity solutions, which might eventually provide holographic duals of the Euclidean theories on the S-brane worldvolumes. As a warm up in section 5.1 we describe the S0-brane in $\mathrm{D}=4$ Einstein-Maxwell gravity. In 5.2 we then find a solution corresponding to the SM5-brane in $\mathrm{D}=11$. These have singularities along the light cones. In section 6 we discuss the issue of the singularities and how they might potentially be resolved. Finally section 7 concludes with a brief summary.

This work was in part inspired by the works of Hull [8,9], who proposed a so-called

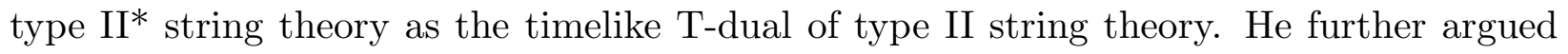
that the $\mathrm{II}^{*}$ theory contains BPS, spacelike D-branes holographically dual to de Sitter

space. The situation where all coordinates obey Dirichlet boundary conditions (the SD(1)-brane) was discussed in the past in order to introduce hard scattering behavior into string amplitudes [15,17] and define off-shell string amplitudes [18,19.

Very recent interesting and possibly related work on time-dependent string backgrounds includes [20,21].

\section{S-branes in $\mathrm{D}=4$ Scalar Field Theory}

\subsection{An S2-brane Domain Wall}

We start with a simple example of a scalar field with the double well potential

$$
V(\phi)=\left(\phi^{2}-a^{2}\right)^{2}
$$

with vacua at $\phi=\phi_{ \pm}= \pm a$. We further weakly couple the scalar field to some form of massless radiation. This theory has ordinary 2-brane domain wall solutions which interpolate between the two vacua.

$\mathrm{S} 2-$ brane solutions can also be constructed as follows. Impose the initial data at $t=0$

$$
\phi(\vec{x}, 0)=0, \quad \dot{\phi}(\vec{x}, 0)=v
$$

where $v$ is a small positive constant. This corresponds to a scalar field about to fall off the local maximum at the origin. As the system evolves forward in time, the scalar field will 
dissipate energy into radiation, oscillating about and decaying towards the $\phi_{+}$vacuum. On the other hand, since the initial conditions are invariant under $\phi \rightarrow-\phi$ together with $t \rightarrow-t$, evolving the system into the past leads to a similar configuration asymptoting to the $\phi_{-}$vacuum.

The full evolution of the system can be described as follows. At $t=-\infty$, the scalar field is near $\phi=\phi_{-}$. Finely tuned radiation conspires to excite the scalar field and push it to the top of barrier. It then falls over the other side, dissipating its energy into more radiation and decaying towards the $\phi_{+}$vacuum. Because $\phi \rightarrow \pm a$ for $t \rightarrow \pm \infty$ (rather than $x \rightarrow \pm \infty$ ), this is a spacelike (rather than timelike) 2-brane.

One could also choose to specify initial data corresponding to $\phi$ perched at its maximum along the null hypersurface $t=x$ (together with vacuum initial data at $t+x \rightarrow-\infty, \quad t>x)$. This leads to an N2-brane.

\subsection{An S1-brane Vortex}

Now consider a complex scalar with the Mexican hat potential

$$
V(\phi)=\left(\phi^{*} \phi-a^{2}\right)^{2}
$$

This has the usual global cosmic string (timelike 1-brane) solutions. S1-brane solutions arise from $e . g$. the initial data

$$
\phi(x, y, z, 0)=a \tanh z, \quad \dot{\phi}(x, y, z, 0)=i v e^{-z^{2}} .
$$

In the far future, after dissipating energy into radiation, the scalar will settle down into a configuration with $|\phi|=a$, but with $\phi=-a$ at $z=-\infty$ interpolating to $\phi=a$ at $z=\infty$ clockwise in the upper half plane. The symmetry of (2.4) under $t \rightarrow-t, \phi \rightarrow \phi^{*}$ then implies that in the far past, the configuration originated in the vacuum with $\phi=-a$ at $z=-\infty$ interpolating $\phi=a$ at $z=\infty$ counterclockwise in the lower half plane. The scalar field makes one full circle about the origin as the asymptotic circle in the $(z, t)$ plane is traversed.

3 Strictly speaking in this case the full scalar plus radiation cannot fully settle down into its ground state because the S-brane fills space and there is no asymptotic region for the radiation to dissipate into. We shall not dwell upon this feature because it does not occur in the higher codimension branes discussed below which are our real interest. 
This configuration can be described as finely tuned radiation which comes in from $z= \pm \infty$, and then is fully absorbed by the scalar field. This excites the scalar field to the top of the bump. It subsequently rolls down the other side of the bump, emitting radiation as it goes.

This construction, as well as that of the previous subsection, have obvious generalizations to both higher spacetime dimension and higher codimension.

\subsection{Charged S-branes}

We wish to consider the possibility of adding adding axion charge to the 1-brane, associated to the three-form field strength $H=d B$. This is accomplished via the coupling

$$
g \int B \wedge d j
$$

where $g$ is a coupling constant and $j$ is the Klein-Gordon one form

$$
j=\frac{i}{4 \pi a^{2}}\left(\phi^{*} \partial_{\mu} \phi-\phi \partial_{\mu} \phi^{*}\right) d x^{\mu}
$$

For the timelike 1-brane, this gives an axion field strength which falls of like $\frac{1}{r}$ away from the string. The axion charge is defined by the integral of $* H$ over a spatial contour encircling the string.

The case of a spacelike 1-brane following from (2.4) is less familiar. On distances large compared to the thickness of the brane, the axion field has a source term given by

$$
d * H=g \delta^{2}\left(t^{+}, t^{-}\right) d t^{-} \wedge d t^{+}
$$

where $t^{ \pm}=t \pm z$. This equation is solved by

$$
H=d x \wedge d y \wedge d \psi\left(t^{+}, t^{-}\right)
$$

where $\psi$ obeys the two-dimensional wave equation with source

$$
2 \partial_{+} \partial_{-} \psi=g \delta^{2}\left(t^{+}, t^{-}\right)
$$

In terms of advanced and retarded Green functions, a time symmetric solution is

$$
\psi\left(t^{+}, t^{-}\right)=\frac{g}{4}\left(G_{\mathrm{adv}}\left(t^{+}, t^{-} ; 0,0\right)+G_{\mathrm{ret}}\left(t^{+}, t^{-} ; 0,0\right)\right) .
$$


Explicitly

$$
\begin{aligned}
G_{\text {ret }}\left(t^{+}, t^{-} ; 0,0\right) & =\Theta\left(t^{+}\right) \Theta\left(t^{-}\right), \\
G_{\text {adv }}\left(t^{+}, t^{-} ; 0,0\right) & =\Theta\left(-t^{+}\right) \Theta\left(-t^{-}\right), \\
\psi\left(t^{+}, t^{-}\right) & =\frac{g}{4} \Theta\left(t^{+} t^{-}\right),
\end{aligned}
$$

$H$ has support on the light cone of the S1-brane

$$
H=\frac{g}{4} d x \wedge d y \wedge\left(d t^{+} \delta\left(t^{+}\right) \epsilon\left(t^{-}\right)+d t^{-} \delta\left(t^{-}\right) \epsilon\left(t^{+}\right)\right) .
$$

It is easy to see that the integral of $* H$ around a contour encircling the origin in the $\left(t^{+}, t^{-}\right)$plane gives a nonzero charge. Of course homogeneous solutions of (2.9) (such as $\left.G_{\text {adv }}-G_{\text {ret }}\right)$ may also be added to (2.11).

This construction can also be generalized to other (co)dimensions. For example, the S2-brane of section 2.1 can be coupled to a 4 -form field strength $F=d C^{(3)}$ via the interaction

$$
\int d \phi \wedge C^{(3)}
$$

However the nature of the field strength around the brane depends qualitatively on whether the codimension is even or odd. For example consider an S0-brane in $\mathrm{D}=4$ which has codimension three. This can be coupled to a Maxwell field, which obeys

$$
d F=0, \quad d^{\dagger} F=d z \delta(t) \delta(x) \delta(y),
$$

corresponding to an S0-brane extended in the z-direction. These equations have the solution

$$
F=\operatorname{Re}\left(d z \wedge d \frac{1}{\sqrt{t^{2}-x^{2}-y^{2}-i \epsilon}}\right) .
$$

$F$ is real and supported inside the past and future light cones of the brane. Note that this differs from (2.12) which is supported only on the light cone. In general odd (even) codimension solutions will be supported in (on) the light cone.

An interesting physical realization of a charged S-brane can be given as follows. A $_{\text {An }}$ electric current in a wire is purely spatial: there is no $j_{0}$ component because the charge density vanishes. However if the current persists indefinitely it is not localized in time and so does not correspond to an S-brane. To get time localization the current flow should stop and start. To arrange this, consider initial data at $t=0$ with no electromagnetic fields, and

4 We are grateful to N. Toumbas for this example. 
the electrons in the wire all moving uniformly to the right. The electrons will subsequently decelerate, and eventually come to rest, due to resistance in the wire and electromagnetic radiation produced by their motion. Evolving backward in time from $t=0$, one arrives at an initial state of incoming radiation with no current in the wire. The full picture is roughly that finely tuned incoming radiation momentarily excites a current in the wire, which decays to outgoing radiation. The charge measured by the integral of $* F$ over the $S^{2}$ is given by the number of electrons which cross any three-dimensional ball whose boundary is the $S^{2}$.

\subsection{R-symmetry}

In the last subsection, massless field solutions were constructed on scales large compared to the S-brane thickness by solving a wave equation with with delta function support on the brane. These solutions are invariant under the transverse Lorentz transformations which leave the location of the brane fixed. For an Sp-brane in D spacetime dimensions, this is an $S O(D-p-2,1)$ "R-symmetry".

However on shorter scales, the exact solutions do not have this R-symmetry, 5 as can be seen directly from the scalar field initial data presented in section 2.2. Anything which is localized to a nonzero finite width region in both space and time can not have a Lorentzian symmetry (that would require localization along the light cone), and there is no Lorentz invariant regularization of the light cone singularity.6 The best exact symmetry one can hope for is $S O(D-p-2)$ spatial rotations.

It is conceivable that this might be describable as spontaneous R-symmetry breaking in an S-brane field theory.

\section{S-branes in String Theory}

In this section we argue that spacelike p-branes appear in string theory.

5 Codimension one branes are an exception as the symmetry is trivial.

6 We are grateful to L. Motl for discussions on this point. 


\subsection{S-branes as Tachyon Solitons}

Let us start with an unstable D3-brane in IIA string theory. This has a worldvolume tachyon $T$ with a potential roughly of the form (2.1). A static unstable D3-brane corresponds to the tachyon perched at the top of the potential. The theory at the bottom of the potential is presumed to correspond to the closed string vacuum and have no propagating open string modes. The decay proceeds when the tachyon slips off the top of the hill. The tachyon is coupled to closed string modes and as it rolls off of the hill its energy is radiated off to infinity in outgoing closed string modes.

As argued by Sen [22] [10], the stable D2-brane is a tachyon kink solution which interpolates between the two vacua $\left(T_{ \pm}= \pm a\right.$ in (2.1) $)$ on a spacelike trajectory. This has nonzero RR charge due to a threebrane worldvolume coupling [10]

$$
\int d T \wedge C_{R R}^{(3)}
$$

as in (2.13). The S2-brane can then be constructed as a timelike rather than spacelike kink. Impose initial data at $t=0$, exactly as in (2.2) with $T=0$ and $\dot{T}>0$. The tachyon will roll off the hill towards the $T_{+}$vacuum. As it rolls the energy will be radiated off to infinity in closed string modes and $T$ will approach $T_{+}$in the infinite future. Evolving to the past one has a time-reversed configuration in which $T \rightarrow T_{-}$in the infinite past. This results in an S2-brane which carries RR charge due to the coupling (3.1).

Sen also proposed an alternate description of the D2-brane as a vortex in the D4-(antiD4) system. This system contains a complex tachyon with a Mexican hat potential. The RR field couples to the vortex number via the coupling $\int C_{R R}^{(3)} \wedge d j$ where $j=\operatorname{Im} T^{*} d T$, the five dimensional analog of (2.5). Hence the spacelike vortex solutions of section 2.2 become S2-branes carrying RR charge. A similar construction on null initial surfaces gives N2-branes.

It is interesting to ask how "thick" the S-branes are in the time direction. This corresponds to the decay time $t_{\text {decay }}$ of the brane-antibrane pair or the unstable brane. Naively one might guess that $t_{\text {decay }} \sim \frac{1}{g_{s}}$ in string units because the decay proceeds via closed strings which couple with strength $g_{s}$.

We suspect that in fact this is not the case and that rather $t_{\text {decay }} \sim \mathcal{O}(1)$. The reason is that the open string theory is strongly coupled at the bottom of the potential. There are no perturbative open string oscillations whatever about the minimum - it is the closed string vacuum. So it is hard to see how $T$ can pass the minimum, let alone undergo many 
oscillations before settling into vacuum. Rather we suppose that $T$ reaches the minimum in a time of order the string time, at which point all excitations can be described as some kind of closed string mode.

One might also imagine that the S-brane can be made arbitrarily thick by making $v$ very small. However in this case quantum fluctuations become important and push $T$ off of the top in a time of order one in string units [23].

\subsection{S-branes within Branes as Evanescent Sphalerons}

Ordinary branes within branes can often be described in terms of smooth gauge field configurations. For example a 0-brane in a pair of 4-branes can be described as an SU(2)

gauge instanton. There is a coupling $\int C_{R R}^{(1)} \wedge \operatorname{tr} F \wedge F$, which implies that the configuration carries the RR charge appropriate to a $D 0$-brane.

We wish to argue that S-branes within branes can have a similar description. While the relevant solutions are more complicated than the tachyon kinks of the previous subsection, the discussion is not plagued by the uncertainties in the tachyon effective Lagrangian. In particular we consider the S(-1)-brane as an event in the D3-brane. What we need is a Lorentzian configuration with a nonzero integral of $\operatorname{tr} F \wedge F$ over some localized spacetime region. We can't take $F=* F$, since that has no real solutions in Minkowski space. Let us take the D3-branes to be slightly separated so that the gauge symmetry is spontaneously broken. Then it is known that there are noncontractible loops in the three-dimensional configuration space which begin and end at the vacuum [24]. However the initial and final vacua have different winding numbers and correspondingly the sequence of configurations has a nonzero value of $\int \operatorname{tr} F \wedge F$. This path passes through a saddle point where the energy takes it maximum value. This saddle point is a static but unstable solution of the equations of motion known as a sphaleron [25] 26].

Now let us specify initial data at $t=0$ for a Lorentzian solution corresponding to sphaleron with a small initial velocity pushing it toward the higher winding vacuum. As time evolves to the future, the sphaleron slides down the hill, energy is radiated off the brane into closed string modes, and the gauge field settles into its vacuum state. Going backwards in time, the system approaches the lower-winding vacuum. Altogether the spacetime configuration traverses the noncontractible loop in the configuration space and 
has integral value of $\operatorname{tr} F \wedge F$. 目 The worldvolume coupling $\int C_{R R}^{(0)} \operatorname{tr} F \wedge F$ then implies that this Lorentzian configuration carries the same RR charge as a Euclidean D-instanton.

When the branes are coincident the gauge symmetry is not broken and such configurations still exist. However it appears that their energy can be made arbitrarily small by spreading them over a large region of space.

We wish to emphasize that these configurations are in no sense the analytic continuation of the usual $\mathrm{SU}(2)$ instanton solutions. These latter have complex values of the fields and are not admissible as real solutions in Minkowski space.

The $g_{s} \rightarrow 0$ limit of these configurations is rather different than that of the tachyon kink S-branes. At $g_{s}=0$ the Yang-Mills-Higgs theory on the D-brane completely decouples from the closed string sector. One then has scattering configurations with nonzero $\operatorname{tr} F \wedge F$ of the type discussed [14] which will source the RR charge. The question of exactly where the RR charge is localized is then rather subtle although likely largely answered in the work of [14]. We will not try to address it herein.

\subsection{NS S-branes}

Duality relates all types of branes and hence suggests that branes carrying NS charge can also be spacelike. In fact the type I / heterotic 5-brane can be described as an instanton in $S O(32)$ or $E 8$. As such its spacelike configuration might be described by the evanescent sphalerons of the preceding subsection. However here, because there is no extra region for the energy to leak off into, we encounter the subtleties described in [14].

\section{Spacelike D-branes}

In the previous section time-dependent solutions of string theory corresponding to spacelike branes were described. They are spacelike counterparts of Sen's solitonic description of the usual D-branes and in particular carry the same RR charges. It is natural to suppose that in string perturbation theory they can be described by an SD-brane - i.e. a conformal field theory in which the timelike worldsheet coordinate $X^{0}$, as well as $8-p$ spacelike coordinates, obey Dirichlet boundary conditions. In the null case one takes $X^{+}$ to obey Dirichlet boundary conditions, but we defer this case to later consideration.

7 A cogent discussion of this type of configuration is given in [14]. Our system is however a bit simpler in that the leakage of energy off of the brane allows the gauge field to fully approach the vacuum asymptotically.

8 Interestingly, ND-branes can have on-shell propagating open strings on their worldvolumes. 


\subsection{The Boundary State}

The SD-brane acts as a source for massless and massive closed string fields. As is the case for timelike D-branes, these fields can be succinctly described at leading order in $g_{s}$ with the use of a boundary state. The spacelike case will turn out to differ only in a few signs from the timelike case. We refer the reader to [27], whose conventions we follow, for details of the latter. The bosonic part of a boundary state which imposes the boundary conditions of a spacelike $p$ brane is given by imposing Dirichlet boundary condition $X^{a}=y^{a}$ on the first $9-p$ coordinates $X^{a}, a=0,1, \cdots, 8-p$ and Neumann boundary conditions on the last $p+1$ coordinates $X^{i}, i=9-p, \cdots, 9$.

$$
\begin{aligned}
\left(\alpha_{n}^{\mu}+O^{\mu}{ }_{\nu} \bar{\alpha}_{-n}^{\nu}\right)|B, \eta\rangle & =0, & & \mu=0,1, \cdots, 9 \\
\left(\psi_{r}^{\mu}-i \eta O^{\mu}{ }_{\nu} \bar{\psi}_{-r}^{\nu}\right)|B, \eta\rangle & =0, & & \mu=0,1, \cdots, 9 \\
\left(q^{a}-y^{a}\right)|B, \eta\rangle & =0, & & a=0,1, \cdots, 8-p \\
p^{i}|B, \eta\rangle & =0, & & i=9-p, \cdots, 9
\end{aligned}
$$

where $O^{\mu \nu}=\operatorname{diag}\left(-\eta^{a b}, \delta^{i j}\right)$ and $\eta= \pm 1$. The solution of these equations is given by

$$
|B, \eta\rangle=N_{p} \exp \left(-\sum_{n} \frac{1}{n} O_{\mu \nu} \alpha_{-n}^{\mu} \bar{\alpha}_{-n}^{\nu}+i \eta \sum_{r} O_{\mu \nu} \psi_{-r}^{\nu} \bar{\psi}_{-r}^{\nu}\right) \int d^{p+1} k e^{i k_{a} y^{a}}|0, \eta\rangle,
$$

Here we did not display the ghost part of the boundary state which is the same as for the standard timelike branes, and both the ground state and oscillator moding differ between R-R and NS-NS sectors. The zero mode part in the NS-NS and R-R sector are given by

$$
\begin{aligned}
|0, \eta\rangle_{N S} & =-i\left|k^{a}, k^{i}=0\right\rangle, \\
|0, \eta\rangle_{R} & =\left(C \Gamma^{9-p \cdots 9} \frac{1+i \eta \Gamma^{11}}{1+i \eta}\right)_{\alpha \beta}\left|\alpha \otimes \bar{\beta}, k^{a}, k^{i}=0\right\rangle .
\end{aligned}
$$

We assume that the normalization $N_{p}$ is the same as in the timelike case, namely

$$
N_{p}=2^{2-p} \pi^{\frac{7}{2}-p} \alpha^{\frac{3-p}{2}}
$$

The D-brane boundary states are then given by the GSO projection invariant combinations $|B, \eta=+1\rangle_{N S}-|B, \eta=-1\rangle_{N S}$ and $|B, \eta=+1\rangle_{R}+|B, \eta=-1\rangle_{R}$ for the NS-NS and $\mathrm{R}-\mathrm{R}$ sector respectively.

A state in the first quantized Hilbert space of a string can be interpreted as a classical string field configuration describing massive and massless closed string fields in spacetime. 
A solution $\mid \Phi>$ of the linearized equation is given in terms of the boundary state via the relation 28] 27]

$$
\left|\Phi>=\frac{1}{L_{0}+\bar{L}_{0}-a}\right| B>,
$$

where $a=1,0$ in the NS-NS and R-R sector respectively. (4.5) is ambiguous because the states can be on shell and the denominator vanish. This is the expected ambiguity corresponding to the freedom to add homogeneous solutions of the equations of motion. For the closed string fields the overlap of the boundary state (4.2) can be related to the corresponding supergravity solution. For example the NS-NS dilaton, in the normalization of [27] obeys

$$
\partial_{a} \partial^{a} \phi(x)=\langle\phi(x) \mid B(y)\rangle=\frac{3-p}{\sqrt{2}} N_{p} \delta^{p+1}(x-y) .
$$

The ( $\mathrm{p}+1)$-form $\mathrm{R}-\mathrm{R}$ potential is of special interest. In the gauge $d * C_{p+1}=0$ it obeys

$$
\partial_{a} \partial^{a} C_{9-p \cdots 9}(x)=4 N_{p} \delta^{p+1}(x-y) .
$$

Hence for the spacelike branes the small fluctuations around flat space will be solutions of the wave equation with a delta function source in the directions transverse to the worldvolume. The equations (4.6) and (4.7) have the same numerical coefficients as their timelike D-brane counterparts. For a time symmetric solution we use the advanced plus retarded propagator, as in section 2.3. This will be supported within the past and future light cones of the brane. We note that the fields diverge not just at a point in the transverse space (the location of the brane) as in the case of timelike branes but instead along the lightcone in transverse space. This divergence will be further discussed in sections 4.2 and 6 .

The charge of the brane can be defined by an integral of the RR field strength or its dual $* F_{p+2}=d C_{p+1}$ over an 8-p sphere surrounding the brane. This 8-p sphere has both timelike and spacelike components. Evaluating this charge with Gauss's law and using (4.7), we find

$$
\int_{S^{8-p}} * F_{p+2}=4 N_{p}
$$

This is numerically the same result obtained for the charges of ordinary branes defined by integrals over spatial spheres. It also agrees with the charges found in the solitonic description of the branes. This result is the main reason for expecting the solitonic and Dirichlet S-branes represent the same basic object. 


\subsection{Discussion}

Schematically, the linearized closed string field configuration is of the form

$$
\Phi \sim G_{\mathrm{adv}}+G_{\mathrm{ret}}
$$

and has a delta function source on the S-brane worldvolume. Consider the alternate configuration

$$
\tilde{\Phi} \sim G_{\mathrm{adv}}-G_{\mathrm{ret}}
$$

This obeys the linearized field equations without a delta function source anywhere. Furthermore $\tilde{\Phi}$ agrees exactly with $\Phi$ in the past of the brane.

This raises an important question: how do the incoming closed strings know whether or not to make an S-brane? A possible answer to this question can be found in the tachyon soliton description of the S-brane. This crucially involved the open string tachyon field. However this field cannot be seen in any order in perturbation theory about the closed string vacuum which sits at the minimum of the tachyon potential. The open strings are hidden non-perturbative degrees of freedom in the closed string vacuum. Therefore we would not have expected to see them in a perturbative description of the incoming state as an excitation of the closed string vacuum. In order to distinguish those incoming state which do and do not form S-branes, we need a much finer description than that supplied by the boundary state.

A second issue concerns the R-symmetry . The boundary state is invariant under the $S O(8-p, 1)$ Lorentz transformations transverse to the branes. As discussed in section 2.4, this cannot be an exact symmetry on scales of order the brane thickness, which we expect to be $\sqrt{\alpha^{\prime}}$ times a power of the string coupling. Hence higher corrections should not preserve this symmetry.

This observation is related to brane energy. 9 The stress energy density of the brane itself has nonzero components

$$
T_{i j} \sim \frac{1}{g_{s}} \delta_{i j} \prod_{a=0}^{p} \delta\left(x^{a}-q^{a}\right) .
$$

This form is largely required by the R-symmetry of the leading order description. It is easy to check that it is conserved. (4.11) gives a positive energy density in any Lorentz frame

9 We are grateful to G. Horowitz and E. Verlinde for discussions on this topic. 
but does not obey the dominant energy condition. (4.11) seems to be in contradiction with the tachyon picture, which suggests a nonzero $T_{00}$ of order $\frac{1}{g_{s}}$. However there is an additional contribution to the energy from the closed strings in the boundary state. This gives an energy density which diverges on the light cone. The origin of this divergence is that the boundary state tries to confine strings to a point. A similar divergence in the Coulomb-like energy occurs for ordinary timelike D-branes, and is resolved by higher corrections. In principle the discrepancy in $T_{00}$ in the tachyon soliton and the D-brane description of S-branes could also be resolved by higher corrections which impart a finite width to the brane. Hence the resolution of this singularity should be seen at the same time as the breaking of the R-symmetry. We have not understood how this would work in detail.

We expect that the worldvolume theory will be a kind of Euclideanization of the usual D-brane theory, including a minus sign in the kinetic term for the field corresponding to fluctuations in the $X^{0}$ directions. The $R$-symmetry (which should be a symmetry of the action) will be the group of transverse rotations which is $S O(9-p-1,1)$ rather than $S O(10-p-1)$.

\section{Spacetime Solutions}

In this section we discuss several solutions of the supergravity equations corresponding to S-branes with odd codimension. In principle these might provide holographic duals of the SD-brane field theories. The basic idea pursued here is simply to consider a general ansatz with $I S O(p+1) \times S O(D-p-2,1)$ symmetry. Of course, to to account for spontaneously broken R-symmetry, a less restrictive ansatze is appropriate. (AdS/CFT examples with spontaneously broken R-symmetry are described in [29].) We hope to give a more complete discussion of the solutions and their properties in future work.

As there is no supersymmetry the solutions are considerably more complex than their timelike partners. 10 The even codimension case is qualitatively different because at linear order the massless fields are supported on (rather than in) the S-brane light cone.

10 We note that naive analytic continuation of the familiar timelike p-brane solutions leads to a complex field strength. 


\subsection{An S0-brane in $D=4$}

In this subsection we consider a simple solution of $\mathrm{D}=4$ Einstein-Maxwell gravity corresponding to a charged S0-brane. This could be relevant to a string compactification with suppressed scalar moduli. The Einstein-Maxwell action is

$$
\int d^{4} x \sqrt{-g}\left(R-F^{2}\right) .
$$

In the absence of gravity, the solution corresponding to a charged S0-brane was given in (2.15), and has an $S O(2,1) \times R$ symmetry. It is natural therefore, when including the gravitational back reaction, to impose this symmetry. We accordingly consider the ansatz

$$
\begin{gathered}
d s^{2}=-\frac{d \tau^{2}}{\lambda^{2}}+\lambda^{2} d z^{2}+R^{2} d H_{2}^{2}, \\
F=Q \epsilon_{2},
\end{gathered}
$$

where $R$ and $\lambda$ are functions of $\tau$ only and $d H_{2}^{2}\left(\epsilon_{2}\right)$ is the line (volume) element on the unit $\mathrm{H}_{2}$ with curvature

$$
R_{a b}=-g_{a b},
$$

for $a, b=0,1$. It is easy to check that (5.3) obeys the Maxwell equations $d F=d * F=0$ as well as

$$
F^{2}=\frac{2 Q^{2}}{R^{4}} .
$$

It remains to determine $R$ and $\lambda$ from the Einstein equations. One finds

$$
\begin{aligned}
R_{a b} & =\frac{g_{a b}}{R^{2}}\left(\left(R R^{\prime} \lambda^{2}\right)^{\prime}-1\right) \\
& =\frac{1}{2} g_{a b} F^{2}, \\
R_{z z} & =\frac{g_{z z}}{R^{2}}\left(R^{2} \lambda \lambda^{\prime}\right)^{\prime} \\
& =-\frac{1}{2} g_{z z} F^{2}, \\
R_{\tau \tau} & =\frac{g_{\tau \tau}}{R^{2}}\left(R^{2}\left(\lambda \lambda^{\prime}\right)^{\prime}+2 R \lambda\left(\lambda R^{\prime}\right)^{\prime}\right) \\
& =-\frac{1}{2} g_{\tau \tau} F^{2},
\end{aligned}
$$

where ${ }^{\prime}$ denotes differentiation with respect to $\tau$. Equating the two expressions for $F^{2}$ gives

$$
\left(R^{2} \lambda^{2}\right)^{\prime \prime}=2 .
$$


Integrating twice gives

$$
R^{2}=\frac{\tau^{2}-\tau_{0}^{2}}{\lambda^{2}}
$$

$\tau_{0}^{2}$ is a (possibly negative) integration constant and we have suppressed the second integration constant corresponding to shifts of $\tau$. Substituting (5.8) and (5.5) into the second equation of (5.6) yields

$$
\left(\left(\tau^{2}-\tau_{0}^{2}\right)\left(\ln \lambda^{2}\right)^{\prime}\right)^{\prime}=-\frac{2 Q^{2} \lambda^{2}}{\tau^{2}-\tau_{0}^{2}}
$$

Defining the new variable $t$ by

$$
\tau=-\tau_{0} \operatorname{coth} \tau_{0} t
$$

so that

$$
\left(\tau^{2}-\tau_{0}^{2}\right) \partial_{\tau}=\partial_{t}
$$

Then (5.9) becomes simply

$$
\partial_{t}^{2} \ln \lambda^{2}=-2 Q^{2} \lambda^{2} .
$$

The solution is

$$
\lambda^{2}=\frac{\alpha^{2}}{Q^{2}} \operatorname{sech}^{2} \alpha t,
$$

where $\alpha$ is an integration constant. The third equation of (5.6) then requires $\alpha=\tau_{0}$, and hence

$$
\lambda^{2}=\frac{\tau_{0}^{2}}{Q^{2}} \frac{\tau^{2}-\tau_{0}^{2}}{\tau^{2}}, \quad R^{2}=\frac{Q^{2} \tau^{2}}{\tau_{0}^{2}}
$$

The line element is

$$
d s^{2}=-\frac{Q^{2}}{\tau_{0}^{2}} \frac{\tau^{2}}{\tau^{2}-\tau_{0}^{2}} d \tau^{2}+\frac{\tau_{0}^{2}}{Q^{2}} \frac{\tau^{2}-\tau_{0}^{2}}{\tau^{2}} d z^{2}+\frac{Q^{2} \tau^{2}}{\tau_{0}^{2}} d H_{2}^{2} .
$$

The asymptotic region with $R \rightarrow \infty$ is at $\tau \rightarrow \pm \infty$ and the metric behaves as

$$
d s^{2} \rightarrow-\frac{Q^{2}}{\tau_{0}^{2}} d \tau^{2}+\frac{\tau_{0}^{2}}{Q^{2}} d z^{2}+\frac{Q^{2} \tau^{2}}{\tau_{0}^{2}} d H_{2}^{2},
$$

and is asymptotically locally flat. Near the brane at $\tau \rightarrow \tau_{0}$ one has, defining $t^{2}=\tau^{2}-\tau_{0}^{2}$

$$
d s^{2} \rightarrow-\frac{Q^{2}}{\tau_{0}^{2}} d t^{2}+\frac{\tau_{0} t^{2}}{Q^{2}} d z^{2}+2 Q^{2} d H_{2}^{2}
$$

\subsection{An S5-brane in $D=11$}

The bosonic action of eleven dimensional supergravity is given by (dropping the WZ term which will not be important in the following).

$$
S=\int d^{11} x \sqrt{-g}\left(R-\frac{1}{2 \cdot 4 !} F^{2}\right)
$$


The equation of motion is given by

$$
R_{\mu \nu}=\frac{1}{2 \cdot 3 !} F_{\mu \lambda \rho \sigma} F_{\nu}^{\lambda \rho \sigma}-\frac{1}{6 \cdot 4 !} g_{\mu \nu} F^{2}
$$

An ansatz 11 which simplifies the equations of motion is

$$
d s^{2}=-e^{-4 f+8 g} d t^{2}+e^{2 f} d x_{6}^{2}+e^{2 g-4 f} d H_{4}^{2},
$$

where $d H_{4}$ is the metric on a hyperbolic space with negative curvature, and

$$
* F=h(t) d t \wedge d x_{1} \wedge \cdots \wedge d x_{6} .
$$

Solving $d F=0$ leads to

$$
h(t)=e^{12 f} q
$$

With this ansatz $(5.19)$ becomes

$$
\begin{aligned}
f^{\prime \prime}+\frac{q^{2}}{6} e^{12 f} & =0, \\
g^{\prime \prime}-3 e^{6 g} & =0, \\
-18 f^{\prime} f^{\prime}+12 g^{\prime} g^{\prime}+2 f^{\prime \prime}-4 g^{\prime \prime}-\frac{q^{2}}{6} e^{12 f} & =0 .
\end{aligned}
$$

It is easy to see that the system of equations (5.23) is equivalent to the following two first oder equations.

$$
g^{\prime} g^{\prime}-e^{6 g}=c, \quad f^{\prime} f^{\prime}+\frac{q^{2}}{36} e^{12 f}=\frac{2 c}{3},
$$

where $c$ is a positive integration constant. The solutions of (5.24) are easily obtained by integration

$$
\begin{aligned}
& f(t)=\frac{1}{12} \ln \left(\frac{c}{\cosh ^{2}\left(2 \sqrt{6 c}\left(t-t_{0}\right)\right)}\right)-\frac{1}{12} \ln \left(q^{2} / 24\right), \\
& g(t)=\frac{1}{6} \ln \left(\frac{c}{\sinh ^{2}\left(3 \sqrt{c}\left(t-t_{1}\right)\right)}\right) .
\end{aligned}
$$

The integration constant $c$ can be eliminated by rescaling $t \rightarrow t / \sqrt{c}$ and $x_{i} \rightarrow x_{i} / c^{\frac{1}{12}}$. Furthermore we are free to set $t_{1}=0$ by a shift. This leaves one constant $t_{0}$. The metric takes the form

$$
\begin{aligned}
d s^{2}= & -\left(\frac{q^{2}}{24}\right)^{\frac{1}{3}} \frac{\left(\cosh \sqrt{24}\left(t-t_{0}\right)\right)^{\frac{2}{3}}}{(\sinh 3 t)^{\frac{8}{3}}}\left(-d t^{2}+(\sinh 3 t)^{2} d H_{4}^{2}\right) \\
& +\left(\frac{q^{2}}{24}\right)^{-\frac{1}{6}} \frac{1}{\left(\cosh \sqrt{24}\left(t-t_{0}\right)\right)^{\frac{1}{3}}} d x_{6}^{2} .
\end{aligned}
$$

11 Similar solutions were obtained in 30] 313] 33] 34. 
The asymptotic region is at $t \rightarrow 0$ where the radius of the $H_{4}$ diverges. Defining $u=t^{-1 / 3}$, near $t=0, u=\infty$ the metric becomes

$$
d s_{t \rightarrow 0}^{2} \sim-\left(\frac{q^{2}}{216}\right)^{\frac{1}{3}}\left(\cosh \sqrt{24} t_{0}\right)\left(-d u^{2}+u^{2} d H_{4}^{2}\right)+\left(\frac{q^{2}}{24}\right)^{-\frac{1}{6}} \frac{1}{\left(\cosh \sqrt{24} t_{0}\right)^{\frac{1}{3}}} d x_{6}^{2}
$$

which is locally flat space in Rindler coordinates. The Ricci scalar behaves as

$$
R=8\left(\frac{3}{q^{2}}\right)^{\frac{1}{3}}\left(\frac{\sinh (3 t)}{\cosh \left(2 \sqrt{6}\left(t-t_{0}\right)\right)}\right)^{\frac{8}{3}}
$$

The large $t$, near-brane behavior is given by

$$
\begin{aligned}
d s^{2} \sim & -\left(\frac{q^{2}}{24}\right)^{\frac{1}{3}} \exp \left(\left(-8+4 \sqrt{\frac{2}{3}}\right) t-4 \sqrt{\frac{2}{3}} t_{0}\right) d t^{2}+\left(\frac{q^{2}}{24}\right)^{-\frac{1}{6}} \exp \left(-2 \sqrt{\frac{2}{3}}\left(t-t_{0}\right)\right) d x_{6}^{2} \\
& +\left(\frac{q^{2}}{24}\right)^{\frac{1}{3}} \exp \left(\left(4 \sqrt{\frac{2}{3}}-2\right) t-4 \sqrt{\frac{2}{3}} t_{0}\right) d H_{4}^{2}+\mathcal{O}(1) .
\end{aligned}
$$

Even though the Ricci scalar tends to zero in this region the geometry is singular because for example the coefficient of $d x_{6}^{2}$ vanishes.

\section{Singularities}

Singularities have been encountered in several related places in our discussion. In constructing the profile of the linearized massless fields sourced by an S-brane, one finds singularities along the light cone of the brane. This divides the spacetime into three regions: the interior of the future brane lightcone, the interior of the past brane lightcone, and the spacelike separated region. Related singularities arose in construction of the full supergravity solutions in section 5 .

We envision four possible fates of these singularities in the full theory:

(i) The singularity is smoothed out by stringy effects which are nonperturbative in $\alpha^{\prime}$. This is the case for the Coulomb-like singularities of ordinary D-branes. It is suggested by the tachyon soliton picture, in which the source for the various closed string fields are spread out over a region of size of order $\sqrt{\alpha}$. As discussed in 2.4 and 4.2 this resolution should be accompanied by breaking of the Lorentzian R-symmetry.

(ii) The singularity is smoothed out by stringy effects which are nonperturbative in $g_{s}$. 
(iii) The maximal extension of the future and past lightcone regions are geodesically complete but do not include one another or the spacelike region. Something like this occurs for example for the ordinary D3 solutions. It also occurs for Hull's SD3-solutions of the $\mathrm{II}^{*}$ theory, for which the near brane region is $\mathrm{dS}_{5}$.

(iv) The singularities are intrinsically "bad" and the S-brane configurations herein should not be considered as fully consistent solutions of string theory.

\section{Summary}

We have shown that ordinary field theories contain time-dependent topological solutions corresponding to S-branes, or branes with only spatial dimensions. S-branes can carry charges measured by flux integrals over spheres with both space and time dimensions. It follows from Sen's description of the tachyon action of unstable D-branes that such objects exist in string theory. Furthermore they carry the charges expected of an S-Dirichlet brane, which has a Dirichlet boundary condition in a timelike direction. Light cone singularities are encountered in both the perturbative string and supergravity descriptions of S-branes, which are analogs of the short-distance singularities of ordinary D-branes. The resolutions of these singularities must be understood before the proper role of S-branes in string theory can be determined.

Acknowledgments. It is a pleasure to thank G. Horowitz, S. Minwalla, L. Motl, J. Polchinski, N. Seiberg, S. Shenker, N. Toumbas, C. Vafa and E. Verlinde for useful discussions. This work was supported in part by DOE grant DE-FG02-91ER40655. 


\section{References}

[1] J. Polchinski, "Dirichlet-Branes and Ramond-Ramond Charges," Phys. Rev. Lett. 75, 4724 (1995) hep-th/9510017.

[2] J. Maldacena, "The large $N$ limit of superconformal field theories and supergravity," Adv. Theor. Math. Phys. 2, 231 (1998) [Int. J. Theor. Phys. 38, 1113 (1998)] hepth/9711200].

[3] S. S. Gubser, I. R. Klebanov and A. M. Polyakov, "Gauge theory correlators from non-critical string theory," Phys. Lett. B 428, 105 (1998) hep-th/9802109.

[4] E. Witten, "Anti-de Sitter space and holography," Adv. Theor. Math. Phys. 2, 253 (1998) hep-th/9802150.

[5] A. Strominger, "The dS/CFT correspondence," JHEP 0110, 034 (2001) hepth/0106113].

[6] A. Strominger, "Inflation and the dS/CFT correspondence," JHEP 0111, 049 (2001) hep-th/0110087.

[7] V. Balasubramanian, J. de Boer and D. Minic, "Mass, entropy and holography in asymptotically de Sitter spaces," hep-th/0110108.

[8] C. M. Hull, "Timelike T-duality, de Sitter space, large N gauge theories and topological field theory," JHEP 9807, 021 (1998) hep-th/9806146].

[9] C. M. Hull, "de Sitter space in supergravity and M theory," JHEP 0111, 012 (2001) hep-th/0109213.

[10] A. Sen, "Non-BPS states and branes in string theory," hep-th/9904207.

[11] I. I. Kogan and N. B. Reis, "H-branes and chiral strings," Int. J. Mod. Phys. A 16, 4567 (2001) arXiv:hep-th/0107163.

[12] M. R. Douglas, "Branes within branes," hep-th/9512077.

[13] J. A. Harvey, P. Horava and P. Kraus, "D-sphalerons and the topology of string configuration space," JHEP 0003, 021 (2000) hep-th/0001143.

[14] E. Farhi, J. Goldstone, S. Gutmann, K. Rajagopal and R. J. Singleton, "Fermion production in the background of Minkowski space classical solutions in spontaneously broken gauge theory," Phys. Rev. D 51, 4561 (1995) hep-ph/9410365.

[15] M. B. Green, "Pointlike Structure And Off-Shell Dual Strings," Nucl. Phys. B 124, 461 (1977).

[16] M. B. Green, "Dynamical Pointlike Structure And Dual Strings," Phys. Lett. B 69, 89 (1977).

[17] M. B. Green, "Pointlike States For Type 2b Superstrings," Phys. Lett. B 329, 435 (1994) hep-th/9403040.

[18] M. B. Green and J. A. Shapiro, "Off-Shell States In The Dual Model," Phys. Lett. B 64, $454(1976)$. 
[19] A. G. Cohen, G. W. Moore, P. Nelson and J. Polchinski, "Semi Off-Shell String Amplitudes," Nucl. Phys. B 281, 127 (1987).

[20] V. Balasubramanian, S. F. Hassan, E. Keski-Vakkuri, and A. Naqvi "A Space-Time Orbifold: A Toy Model for a Cosmological Singularity", hep-th/0202187.

[21] H. Liu, G. Moore and N. Seiberg, to appear.

[22] A. Sen, "SO(32) spinors of type I and other solitons on brane-antibrane pair," JHEP 9809, 023 (1998) hep-th/9808141.

[23] B. Craps, P. Kraus and F. Larsen, "Loop corrected tachyon condensation," JHEP 0106, 062 (2001) hep-th/0105227.

[24] C. Taubes "The Existence of a Nonminimal Solution to the Yang-Mills Higgs Equations on R3", Comm. Math. Phys. 86 (1982) 257; 86 (1982) 299.

[25] N. S. Manton, "Topology In The Weinberg-Salam Theory," Phys. Rev. D 28, 2019 (1983).

[26] F. R. Klinkhamer and N. S. Manton, "A Saddle Point Solution In The Weinberg-Salam Theory," Phys. Rev. D 30, 2212 (1984).

[27] P. Di Vecchia, M. Frau, I. Pesando, S. Sciuto, A. Lerda and R. Russo, "Classical pbranes from boundary state," Nucl. Phys. B 507, 259 (1997) hep-th/9707068, P. Di Vecchia and A. Liccardo, "D-branes in string theory. II," hep-th/9912275.

[28] M. B. Green and M. Gutperle, "Light-cone supersymmetry and D-branes," Nucl. Phys. B 476, 484 (1996) hep-th/9604091.

[29] J. M. Maldacena and C. Nunez, "Towards the large N limit of pure $\mathrm{N}=1$ super Yang Mills," Phys. Rev. Lett. 86, 588 (2001), [hep-th/0008001].

[30] H. Lu, S. Mukherji, C. N. Pope and K. W. Xu, "Cosmological solutions in string theories," Phys. Rev. D 55, 7926 (1997) arXiv:hep-th/9610107.

[31] H. Lu, S. Mukherji and C. N. Pope, "From p-branes to cosmology," Int. J. Mod. Phys. A 14, 4121 (1999) arXiv:hep-th/9612224.

[32] A. Lukas, B. A. Ovrut and D. Waldram, "Cosmological solutions of type II string theory," Phys. Lett. B 393, 65 (1997) arXiv:hep-th/9608195.

[33] A. Lukas, B. A. Ovrut and D. Waldram, "String and M-theory cosmological solutions with Ramond forms," Nucl. Phys. B 495, 365 (1997) arXiv:hep-th/9610238.

[34] C. Grojean, F. Quevedo, G. Tasinato and I. Zavala, "Branes on Charged Dilatonic Backgrounds: Self-Tuning, Lorentz Violations and Cosmology," JHEP 0108, 005 (2001) arXiv:hep-th/0106120. 